\title{
Esquema para la categorización de la peligrosidad de sedimentos de fondo contaminados de sistemas fluviales
}

\author{
Peluso, M. L. ${ }^{1}$, Salibián, A. ${ }^{2}$ y Ronco A. E. ${ }^{1, *}$
}

${ }^{1}$ Centro de Investigaciones del Medio Ambiente, Facultad de Ciencias Exactas, Universidad Nacional de La Plata, 47 y 115, La Plata (1900), Argentina. CONICET. lpeluso@quimica.unlp.edu.ar, cima@quimica.unlp.edu.ar ${ }^{2}$ Departamento de Ciencias Básicas (PRODEA-INEDES), Universidad Nacional de Luján, Casilla de Correo 221, B6700ZBA, Luján, Argentina.

*Corresponding author: cima@quimica.unlp.edu.ar

Received: 04/12/2014

Accepted: 07/10/2015

\section{RESUMEN}

Esquema para la categorización de la peligrosidad de sedimentos de fondo contaminados de sistemas fluviales

Los sedimentos de fondo son receptores de contaminantes y pueden comportarse como fuentes secundarias de contaminación, al ser resuspendidos por procesos naturales o actividades humanas, tales como las operaciones de dragado. En este estudio se utilizaron datos de una caracterización integrada (parámetros fisicoquímicos y toxicológicos) de sedimentos de fondo de sitios contaminados de tributarios del Río de la Plata y su línea costera, para realizar una categorización de la peligrosidad de muestras para ser utilizada en criterios de decisión para disponer materiales provenientes de operaciones de dragado. Se consideraron sedimentos de 27 sitios, sobre los que se determinaron diferentes variables como granulometría, contenido de materia orgánica, sulfuros, metales y plaguicidas. También se evaluaron efectos biológicos por medio de bioensayos de toxicidad (supervivencia y crecimiento de anfípodos), obteniéndose un total de 364 datos de mediciones fisicoquímicas y ecotoxicológicas. Con esta información se realizaron: a) análisis de componentes principales con los datos fisicoquímicos y correlación entre los factores principales y las respuestas biológicas, b) se calcularon dos índices, un índice de calidad de sedimentos el cual incluye datos químicos en relación con valores de referencia, y un índice ecotoxicológico para evaluar la calidad de sedimentos, basado en datos químicos y ecotoxicológicos, que permite categorizar cada sitio con un único valor. Los análisis incluyeron como muestras peligrosas aquellas que mostraron las mayores respuestas tóxicas, principalmente asociadas a la presencia de metales pesados y elevado contenido de sulfuros y materia orgánica.

Palabras claves: Sedimentos contaminados, índice de peligrosidad, ecotoxicología, Hyalella curvispina.

\section{ABSTRACT}

\section{Hazard assessment scheme for ranking contaminated bottom sediments from fluvial systems}

Bottom sediments are receptors of contaminants and could also be secondary sources of pollution. Associated contaminants can be resuspended by natural processes or human activities, such as dredging operations. Data of an integrated characterization (physicochemical and ecotoxicological parameters) of bottom sediments from contaminated sites in tributaries of the Rio de la Plata and its coastline were used to generate hazard criteria for ranking samples for a better management of sediments coming from dredging activities. We used sediment sample data from 27 sites, on which several variables were determined: particle size, content of organic matter, sulfides, metals and pesticides, as well as biological effects assessed by a toxicity test (survival and growth of amphipods). A total of 364 measures were obtained and analyzed: a) principal component analysis was performed and correlations between physicochemical and biological responses were tested, $b$ ) two indexes were calculated: the sediment quality index which includes chemical data in relation to guideline values, and the ecotoxicological index to assess sediment quality based on chemical and ecotoxicological data. Finally, we rank each site with an unique value. The analysis determined as hazardous samples those with the greater toxic response, mainly associated to the presence of heavy metals, high content of sulfides, and organic matter present in the sediments.

Key words: Polluted sediments, hazard index, ecotoxicology, Hyalella curvispina. 


\section{INTRODUCCIÓN}

Los compuestos tóxicos contaminantes que ingresan a los cuerpos de agua superficiales se reparten entre la fase acuosa y la fase particulada, siendo ésta formadora de sedimentos a lo largo del tiempo (Rand et al., 1995; Walker et al., 2006). De esta manera los sedimentos de fondo se comportan como aceptores finales de contaminantes pudiendo actuar como fuentes secundarias de contaminación (Burton, 2002). Los compuestos asociados a los sedimentos pueden ser resuspendidos de forma natural, por procesos físicos o biológicos (bioturbación), o por la actividad humana, tal como frecuentemente ocurre en la actividad de dragado (USEPA, 2000 Sprovieri et al., 2007). Estos mecanismos provocan la removilización y redistribución de los contaminantes a la columna de agua desde los sedimentos (Walker et al., 2006; Hill, 2010).

Para poder tomar decisiones sobre las acciones de remediación en ambientes acuáticos perturbados (eliminación de fuentes de contaminación; dragado de cursos de agua; selección de sitios de disposición de sedimentos provenientes de dragado), se definen niveles guía de calidad para la protección de la biota a compuestos químicos individuales, que son utilizados en la toma de decisiones en planes de gestión ambiental (IADC/CEDA, 1997; CEQG, 2002). Una de las formas más frecuentes de evaluación es la determinación del contenido de contaminantes y la comparación con dichos niveles. Sin embargo, los datos de análisis químicos que determinan las concentraciones totales de contaminantes en una muestra de sedimento podrían estar sobre o subestimando la fracción biodisponible (Alexander, 2000). Una estrategia utilizada entre las herramientas de diagnóstico ambiental, es la realización de bioensayos de toxicidad en laboratorio para evaluar la toxicidad potencial de una matriz contaminada. Los bioensayos de toxicidad con sedimento completo son una herramienta eficaz para evaluar la biodisponibilidad de los contaminantes para la fauna bentónica (Landrum \& Robbins, 1990; Riba et al., 2004). Entre los organismos más utilizados en este tipo de bioensayo se encuentran los anfípodos (Ingersoll et al,
1995; Costa et al., 1998; USEPA, 2000; Correia et al., 2002; Neuparth et al., 2005). En Argentina, en los últimos años se ha venido utilizando el anfípodo Hyalella curvispina, especie distribuida en Sudamérica, como organismo prueba en bioensayos de toxicidad tanto en sedimentos como en matrices acuosas (Giusto \& Ferrari, 2008; Anguiano et al., 2008; García et al., 2010; Mugni et al., 2011; Giusto et al., 2012; Peluso et al., 2011, 2013a). Si bien el estudio de la estructura de las comunidades bentónicas puede presentar mayor sensibilidad a la contaminación que los bioensayos de toxicidad en laboratorio (Hyland et al., 2003; Wenning et al., 2004), es imprescindible contar con sitios de referencia de características similares a los lugares de estudio, ya que la estructura y función de una comunidad bentónica puede ser alterada por numerosos factores naturales, además de la contaminación del sedimento (por ejemplo: granulometría del material, cambios físicos en el agua, recursos limitados o interacciones biológicas y efectos por erosión). Ambos tipos de factores pueden actuar en conjunto generando los cambios observados en las comunidades bentónicas de sitios contaminados.

En este marco conceptual, se realiza un enfoque de diagnóstico integrado, que incluye la utilización de diferentes líneas de evidencia (LDE) para evaluar calidad de sedimentos y potencial sustentabilidad de la biota (Losso et al., 2004; Abessa et al., 2008; McPherson et al., 2008). Una forma de realizar evaluaciones integradas ampliamente difundida es la Triada de Calidad de Sedimentos (sigla en inglés SQT) (Burton, 2002), la cual recurre a diferentes LDE que incluyen, como mínimo, la concentración de contaminantes en el sedimento, la toxicidad asociada y la composición de la comunidad bentónica correspondiente al ambiente en particular.

A partir de diferentes LDEs se han empleado análisis bivariados y multivariados (correlación de Spearman, PCA, NMDS) para evaluar relaciones potenciales entre niveles de exposición (análisis químico) y efectos (bioensayos y estudio de comunidades), para clasificar y/o categorizar sedimentos contaminados (McPherson et al., 2008; Cesar et al., 2009). 
Frecuentemente se genera una controversia en la toma de decisiones sobre el destino de materiales contaminados asociado a su remoción por diversos tipos de obras, tales como el mantenimiento del calado de canales de navegación, mantenimiento de puertos, saneamiento de arroyos y ríos, etc. En este sentido, para evaluar la peligrosidad relativa de los sedimentos de fondo se utilizan índices de categorización, mediante los cuales se logran unificar resultados de bioensayos de toxicidad, junto a información fisicoquímica, en un único valor (Bombardier, 2005; Krebs, 2005).

Los antecedentes de estudios realizados en ríos o arroyos de la Cuenca del Plata, Sudamérica, se han orientado al diagnóstico de calidad, valorando niveles de contaminación y efectos biológicos asociados a diverso tipo de fuentes contaminantes (Ronco et al., 1995; Ronco et al., 2001; Camilión et al., 2003; Ronco et al., 2008; Peluso et al. 2011, 2013a, 2013b) y acciones de dragado (Kreimer et al., 1996), permitiendo contar con información de base para investigar el uso de herramientas a ser utilizadas en la toma de decisiones a nivel regional. Teniendo en cuenta estos antecedentes se propone aplicar dos tipos de abordaje para el análisis de datos sedimentológicos, fisicoquímicos y ecotoxicológicos por medio de análisis multivariado y el uso de índices de calidad de sedimentos para establecer áreas que presenten riesgos ambientales, además de generar herramientas simples y eficaces para su uso en la categorización y toma de decisiones para el manejo y destino de materiales contaminados.

\section{MATERIALES Y MÉTODOS}

\section{Sitios de estudio y datos analizados}

Este estudio utiliza datos sedimentológicos, físico-químicos y ecotoxicológicos de sedimentos de fondo de ríos y arroyos pertenecientes a la región de pampa ondulada de la Cuenca del Plata, en particular en las desembocaduras de arroyos

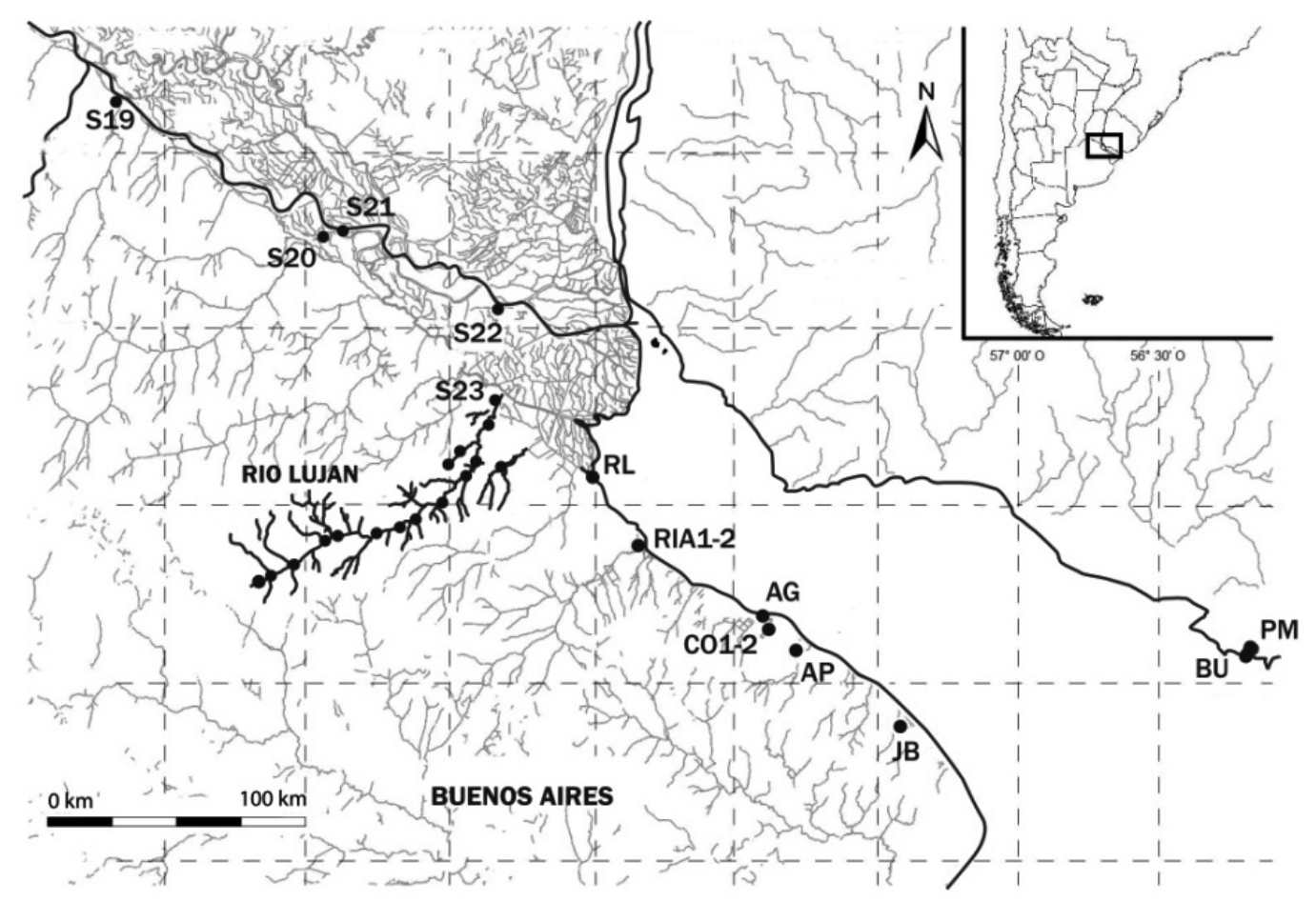

Figura 1. Sitios de estudio. Area of study. 
afluentes de la franja costera sur del Río de la Plata (Ronco et al., 2008; Peluso et al., 2011), a lo largo de la cuenca del Río Luján (Peluso et al., 2013a) y en el tramo medio e inferior del Río Paraná (Peluso et al., 2013b).

Se trabajó con un banco de datos correspondiente a 27 sitios de estudio en los cuales se extrajeron sedimentos de fondo (Fig. 1), que se detallan a continuación:

- Franja costera Río de la Plata: Arroyo Juan Blanco (JB); Arroyo del Pescado (AP); Arroyo el Gato (AG); Riachuelo desembocadura (RIA); Canal Oeste (CO); Puerto de Montevideo (PM); Costa uruguaya antes del Puerto de Montevideo (BU). Estos sitios corresponden a afluentes del Río de la Plata del área metropolitana de Buenos Aires y Gran La Plata en la costa argentina, y el área metropolitana de Montevideo (como principales asentamientos industriales) en la costa uruguaya, los cuales incluyen sitios con una heterogénea carga de contaminantes como los correspondientes a los puertos de Buenos Aires y Montevideo y la zona del Canal Oeste asociado con descargas de refinerías e industrias petroquímicas (Ronco et al., 2008).

- Cuenca Río Luján: Arroyo Durazno (SU-1); Arroyo Leones (SU-2); Puente García (SU-3); Puente 3 de Marzo Mercedes (SU-4); Jáuregui (SM-1); Puente A. Brown Luján (SM-2); Pilar (SM-3); Intersección Ruta 6(SM-4); Arroyo Larena aguas arriba (SM-5) y aguas abajo (SM-6) del complejo industrial Pilar; intersección Acceso Oeste (SL-1); reserva natural Pilar (SL-2); intersección Ruta 9 Escobar (SL-3); Carmel Pilar (SL-4). El Río Luján está ubicado en el noreste de la provincia de Buenos Aires. Las principales fuentes de contaminación provienen de actividad agrícola en la cuenca alta (Andrade, 1986; Guichón et al., 1999) y actividades industriales (efluentes industriales sin tratamiento de complejos industriales Pilar y Luján) en los sectores medio y bajo, además de descargas residuales crudas. Las industrias que tuvieron un mayor crecimiento se relacionan con producción química y petroquímica, además de industrias alimenticias y textiles (Briano et al., 2003).

- Afluentes del Río Paraná: Arroyo Ramallo (S19); Río Arrecifes (S20); Río Areco (S21); Río Paraná de las Palmas (S22); Río Luján (S23), los cuales se ubican en el tramo inferior de la cuenca, correspondiente a Buenos Aires. Los sitios S19, S20 y S23 se encuentran en las desembocaduras de los arroyos y ríos antes mencionados; el S22 corresponde al curso principal del Río Paraná en la localidad de Campana; mientras que el sitio S23 a un sector del Río Luján ubicado al finalizar el tramo medio del mismo (Peluso et al., 2013b). Los tres primeros son cursos que atraviesan zonas netamente agrícolas, mientras que los dos últimos tienen influencia de tipo urbano-industrial.

Para cada muestra se determinaron las siguientes variables: granulometría, materia orgánica, sulfuros, metales y plaguicidas (Tabla 1). Se utilizaron metodologías estandarizadas, las cuales se describen en Ronco et al. (2008) y Peluso et al. (2013a). Además, se realizaron bioensayos de toxicidad sobre sedimento directo con el anfípodo $H$. curvispina obteniéndose respuestas letales (supervivencia) y subletales (crecimiento) en exposiciones de 10 días, cuya metodología se describe en Peluso et al. (2011; 2013a-b). El presente análisis se realizó sobre un total de 364 datos.

\section{Análisis de datos}

\section{Análisis de componentes principales}

Con el objetivo de interpretar los datos obtenidos en la totalidad de las muestras de sedimentos (27 sitios) estudiados, se realizó un análisis multivariado con los resultados de la caracterización fisicoquímica. Las variables incluidas en el análisis fueron las siguientes: materia orgánica (MO), contenido de finos, sulfuros, metales $(\mathrm{Cd}, \mathrm{Cr}, \mathrm{Cu}$, $\mathrm{Fe}, \mathrm{Hg}, \mathrm{Pb}$ y $\mathrm{Zn}$ ) y plaguicidas totales. Se realizó un análisis de componentes principales (ACP), que permite limitar el conjunto de variables que caracterizan fisicoquímicamente las muestras de 
sedimentos a un número reducido de componentes (factores), que expliquen la variabilidad total que presentan las muestras. El criterio de selección de los factores fue que tuvieran valores propios mayores a 1 (Quinn \& Keough, 2002). Posteriormente se procedió, por medio de análisis de regresión y correlación (Zar, 2010), a determinar la relación entre los factores seleccionados y los resultados de los bioensayos de toxicidad en $H$. curvispina expresados como porcentaje de supervivencia y porcentaje de inhibición de crecimiento.

\section{Índices de calidad de sedimentos}

Para realizar una primera clasificación de los sedimentos, las muestras fueron categorizadas utilizando el Índice de Calidad de Sedimentos (SQI de las siglas en inglés) que se calcula sobre la ba-

Tabla 1. Parámetros físico-químicos, contaminantes (metales y plaguicidas totales PMT) y efectos medidos en las muestras de sedimentos analizadas. Physical and chemical parameters, contaminants (metals and totals pesticides PMT) and effects assessed at the studied sediment samples.

\begin{tabular}{|c|c|c|c|c|c|c|c|c|c|c|c|c|c|}
\hline \multirow[t]{2}{*}{ Sitio } & \multicolumn{3}{|c|}{ Propiedades del sedimento } & \multicolumn{6}{|c|}{ Metales $(m g / K g)$} & \multicolumn{2}{|c|}{$\begin{array}{c}\text { Plaguicidas } \\
\quad(\mu \mathrm{g} / \mathrm{Kg})\end{array}$} & \multicolumn{2}{|c|}{ Efectos (\%) } \\
\hline & $\mathrm{MO}(\%)$ & $\begin{array}{c}\text { Finos } \\
(\%)\end{array}$ & Sulfuros & $\mathrm{Cd}$ & $\mathrm{Cu}$ & $\mathrm{Cr}$ & $\mathrm{Hg}$ & $\mathrm{Pb}$ & $\mathrm{Zn}$ & $\mathrm{Fe}$ & PMT & Supervivencia & $\begin{array}{c}\text { Inhibición } \\
\text { Crecimiento }\end{array}$ \\
\hline $\mathrm{JB}^{a}$ & 2.2 & 85.1 & 2.5 & 1.01 & 15.8 & 2.5 & 0.60 & 37.3 & 69.9 & 24301 & 2.7 & 92 & 0 \\
\hline $\mathrm{RL}^{a}$ & 2.8 & 83.4 & 2.5 & $<0.51$ & 7.9 & 20.3 & 0.08 & 33.4 & 44.3 & 16653 & 309.0 & 86 & 52.8 \\
\hline $\mathrm{RIA}^{a}$ & 13.0 & 60.5 & 2923 & 2.43 & 136.1 & 1141.1 & 1.90 & 204.9 & 706.7 & 27585 & 3.3 & 10 & 100 \\
\hline $\mathrm{CO}^{a}$ & 21.5 & 80.9 & 1343 & 3.16 & 55.8 & 2.5 & 6.80 & 124.7 & 387.3 & 34263 & 6.7 & 0 & 100 \\
\hline $\mathrm{AG}^{a}$ & 17.3 & 95.8 & 278 & 1.08 & 32.4 & 27.0 & 0.12 & 54.0 & 97.6 & 6410 & nd & 80 & 39.7 \\
\hline $\mathrm{AP}^{a}$ & 11.6 & 89.8 & 178 & $<0.78$ & 15.6 & 19.4 & 0.04 & 27.2 & 194.6 & 11718 & nd & 88 & 0 \\
\hline $\mathrm{BU}^{a}$ & 37.4 & 51.2 & 550 & $<0.15$ & 31.4 & 40.3 & 3.10 & 15.0 & 9 & 36274 & nd & 82 & 0 \\
\hline $\mathrm{PM}^{a}$ & 62.1 & 95.0 & 1939 & $<0.15$ & 107.1 & 436.1 & 5.40 & 15.0 & 290.7 & 52028 & nd & 0 & 100 \\
\hline SU-1 ${ }^{b}$ & 2.7 & 69.9 & 176 & $<0.15$ & 23.3 & 9.4 & $<0.015$ & 23.6 & 39.0 & 20945 & 31.0 & 34 & 100 \\
\hline SU- $2^{b}$ & 2.9 & 83.7 & 220 & $<0.15$ & 18.8 & 13.3 & $<0.015$ & 24.3 & 32.0 & 26692 & 5.5 & 70 & 34.9 \\
\hline SU- $3^{b}$ & 2.6 & 72.2 & 130 & $<0.15$ & 20.3 & 17.0 & $<0.015$ & 26.2 & 22.0 & 26701 & 9.6 & 68 & 27.2 \\
\hline SU- $4^{b}$ & 10.2 & 70.3 & 215 & $<0.15$ & 126.6 & 807.3 & $<0.015$ & 64.1 & 190.0 & 28750 & 15.9 & 10 & 100 \\
\hline SM- $1^{b}$ & 6.6 & 74.7 & 20 & $<0.15$ & 31.4 & 149.5 & $<0.015$ & 73.3 & 170.0 & 20714 & 3.5 & 58 & 50.9 \\
\hline $\mathrm{SM}-2^{b}$ & 7.7 & 64.0 & 10 & $<0.15$ & 98.8 & 219.7 & $<0.015$ & 57.1 & 196.0 & 24804 & nd & 82 & 0 \\
\hline $\mathrm{SM}-3^{b}$ & 3.5 & 78.7 & 34 & 23.4 & 23.1 & 19.5 & $<0.015$ & 33.0 & 75.0 & 25308 & 9.9 & 84 & 81.1 \\
\hline $\mathrm{SM}-4^{b}$ & 3.6 & 78.9 & 27 & $<0.15$ & 37.2 & 5.8 & $<0.015$ & 34.9 & 145.0 & 20750 & 10.2 & 80 & 0 \\
\hline $\mathrm{SM}-5^{b}$ & 3.8 & 78.9 & 20 & $<0.15$ & 25.1 & 35.7 & $<0.015$ & 24.6 & 68.0 & 28017 & 0.9 & 87 & 0 \\
\hline SM- $6^{b}$ & $\quad 12.1$ & 79.8 & 1409 & $<0.15$ & 466.0 & 51.4 & 11.00 & 104.9 & 751.0 & 32818 & 21.4 & 8 & 100 \\
\hline SL- $1^{b}$ & 3.6 & 69.4 & 310 & $<0.15$ & 24.3 & 40.9 & $<0.015$ & 172.4 & 38.0 & 25550 & nd & 84 & 48.2 \\
\hline $\mathrm{SL}-2^{b}$ & 23.2 & 81.2 & 98 & $<0.15$ & 142.1 & 60.0 & $<0.015$ & 63.8 & 265.0 & 68125 & 18.5 & 66 & 25 \\
\hline $\mathrm{SL}-3^{b}$ & 3.4 & 71.5 & 23 & $<0.15$ & 26.8 & 28.1 & $<0.015$ & 29.1 & 79.0 & 16094 & 31.7 & 90 & 53.3 \\
\hline $\mathrm{SL}-4^{b}$ & 4.3 & 97.5 & 10 & $<0.15$ & 27.3 & 40.9 & $<0.015$ & 31.1 & 98.0 & 25219 & 60.2 & 94 & 43.1 \\
\hline $\mathrm{S} 19^{c}$ & 15.5 & 96.2 & 490 & $<0.15$ & 13.2 & 20.6 & $<0.015$ & 29.1 & 49.4 & 13060 & 27.3 & 92 & 29 \\
\hline $\mathrm{S} 20^{c}$ & 40.0 & 90.4 & 103 & $<0.15$ & 11.6 & 17.2 & $<0.015$ & 23.3 & 40.7 & 12430 & 7.7 & 90 & 41.3 \\
\hline $\mathrm{S} 21^{c}$ & 17.1 & 89.6 & 114 & $<0.15$ & 8.9 & 9.3 & $<0.015$ & 16.3 & 28.5 & 5660 & 1.7 & 88 & 41.7 \\
\hline $\mathrm{S} 22^{c}$ & 21.9 & 59.4 & 17 & $<0.15$ & 13.0 & 19.7 & $<0.015$ & 23.7 & 35.5 & 16790 & nd & 90 & 85.4 \\
\hline $\mathrm{S} 23^{c}$ & 4.4 & 71.2 & - & $<0.15$ & 19.3 & 30.7 & $<0.015$ & 27.2 & 61.7 & 12550 & 31.3 & 88 & 59.2 \\
\hline
\end{tabular}

nd: no detectable

${ }^{a}$ Ronco et al. 2008 y Peluso et al. 2011

${ }^{b}$ Peluso et al. 2013a

${ }^{c}$ Peluso et al. 2013b 
se de valores guía de calidad (VG). Se utilizó el índice propuesto por Grapentine et al. (2002), el cual se detalla a continuación:

$$
\begin{aligned}
F_{1}= & (\text { Variables que exceden } * 100 \\
& \text { el VG/Variables totales })
\end{aligned}
$$

$F_{2}=g m n c / 0,01 * g m n c+0,01$

$g m n c=\frac{\sum(\text { valor concentración } / V G)-1}{\mathrm{~N}^{0} \text { de muestras }}$

donde gmnc es el grado medio de no cumplimiento con el valor guía

$$
S Q I=100-\frac{\sqrt{F_{1}^{2}+F_{2}^{2}}}{1,414}
$$

El valor de concentración/VG indica el grado en que una variable excede su valor guía. Tomando como base la clasificación realizada por los autores del índice, se adoptó el siguiente criterio de clasificación para las muestras de acuerdo a la calidad de sus sedimentos: excelente, valores de SQI entre 95-100; no peligroso, entre 80-94; probablemente peligroso, entre 79-60; peligroso, entre 45-59 y muy peligroso, entre 0-44.

Las variables químicas utilizadas en el cálculo fueron metales $(\mathrm{Cd}, \mathrm{Cr}, \mathrm{Cu}, \mathrm{Fe}, \mathrm{Hg}, \mathrm{Pb}$ y $\mathrm{Zn})$ y contenido total de plaguicidas. Debido a la ausencia de niveles guía de calidad de sedimentos en Argentina, se tomaron los valores correspondientes a los niveles guía de Canadá (CEQG, 2002). Los mismos proponen niveles de referencia para protección de vida acuática asociados a sedimentos, tomando como referencia sedimentos no contaminados (Interim Freshwater Sediment Quality Guideline, ISQG), y niveles con probables efectos sobre el medio ambiente (Probable Effect Level, PEL) (CEQG, 2002).

\section{Índice Ecotoxicológico de Peligrosidad de Sedimentos}

Se desarrolló un índice propio de categorización de la peligrosidad (Índice EPS), que incluye variables químicas (contenido de $\mathrm{Cd}, \mathrm{Cr}, \mathrm{Cu}, \mathrm{Fe}$,
Tabla 2. Peso de los factores y varianza total explicada por los tres componentes principales. Factor loadings and percentage of the total variance explained by the three first components.

\begin{tabular}{lrrr}
\hline & $F 1$ & $F 2$ & $F 3$ \\
\hline Valor propio & 4.184 & 1.573 & 1.200 \\
Varianza acumulada & 38.039 & 52.335 & 63.245 \\
$\mathrm{MO}$ & 0.345 & 0.691 & -0.469 \\
$\mathrm{Sulfuros}$ & 0.865 & -0.026 & -0.090 \\
Finos & -0.198 & 0.586 & 0.360 \\
$\mathrm{Cd}$ & -0.037 & -0.176 & 0.014 \\
$\mathrm{Cu}$ & 0.792 & 0.130 & 0.353 \\
$\mathrm{Cr}$ & 0.625 & -0.419 & -0.295 \\
$\mathrm{Hg}$ & 0.774 & 0.389 & 0.275 \\
$\mathrm{~Pb}$ & 0.679 & -0.526 & 0.073 \\
$\mathrm{Zn}$ & 0.933 & -0.070 & 0.230 \\
$\mathrm{Fe}$ & 0.532 & 0.302 & -0.225 \\
$\mathrm{Plaguicidas}$ totales & -0.212 & -0.061 & 0.668 \\
\hline
\end{tabular}

$\mathrm{Hg}, \mathrm{Pb}, \mathrm{Zn}$ y plaguicidas) y la respuesta biológica de $H$. curvispina obtenida en los bioensayos de toxicidad aguda (efectos letales y subletales). El índice se calculó de la siguiente forma:

$$
I E P S=\frac{\left[\sum(\mathrm{Cm} / \mathrm{Cg}) / n\right]}{\text { Tox }}
$$

donde $\mathrm{Cm}$ corresponde a la concentración medida de cada compuesto o familia en la muestra y $\mathrm{Cg}$ el valor guía correspondiente; $n$ es el número de compuestos analizados y el término Tox corresponde a la respuesta biológica. El IEPS se calculó con el valor guía correspondiente de calidad de sedimentos (ISQG) y el nivel de efecto probable (PEL), además se incluyó un cálculo utilizando los valores del sedimento de referencia utilizado, Arroyo Juan Blanco, considerado un ambiente de bajo impacto por contaminación. Para establecer un valor que exprese la toxicidad y que incluya respuestas letales y subletales se adoptó una clasificación a priori, propuesta por McPherson et al. (2008) con modificaciones, otorgándole valores numéricos para poder establecer el término correspondiente a la respuesta biológica en la fórmula del IEPS.

Las categorías de calidad de los sedimentos fueron establecidas de la siguiente manera: Índice entre 0.01-0.09: no peligroso; entre 0.10-0.99: 
probablemente peligroso; entre 1.00-9.90: peligroso; >10: muy peligroso.

\section{RESULTADOS}

\section{Análisis de componentes principales}

A partir de los resultados obtenidos del ACP se seleccionaron del análisis los tres primeros componentes (F1, F2 y F3), los cuales explicaron el $65 \%$ de la varianza total. El F1 explica el mayor porcentaje de variación y agrupa los sulfuros y los metales $\mathrm{Cu}, \mathrm{Zn}, \mathrm{Cr}, \mathrm{Pb}, \mathrm{Hg}$ y en menor medida $\mathrm{Fe}$, mientras que el F2 presenta valores significativos para la materia orgánica y el contenido de finos (Tabla 2); por otro lado el F3 presentó valores significativos para plaguicidas. La figura 2 muestra el ordenamiento en dos dimen- siones representado por el F1 y F2 obtenidos del PCA para los sitios y variables. El análisis de correlación entre los factores y la respuesta de los organismos prueba en los bioensayos de toxicidad muestra una relación negativa altamente significativa $(r=-0,82 ; p<0,01)$, entre la respuesta como \% de supervivencia (Fig. 3) y el F1. Por otro lado no se encontraron correlaciones entre la supervivencia y los F2 y F3. A su vez la inhibición de crecimiento de $H$. curvispina no se correlaciona significativamente con ninguno de los factores obtenidos mediante el PCA. Estos resultados indicarían que el componente 1 , representado por los metales $\mathrm{Zn}, \mathrm{Cu}, \mathrm{Cr}$ y $\mathrm{Pb}$, junto con los sulfuros, es representativo de la composición de la matriz que genera toxicidad letal en las muestras de sedimento evaluadas.

En la figura 2 se puede observar que el F1 fue el predominante en las muestras RIA, SM-6, PM

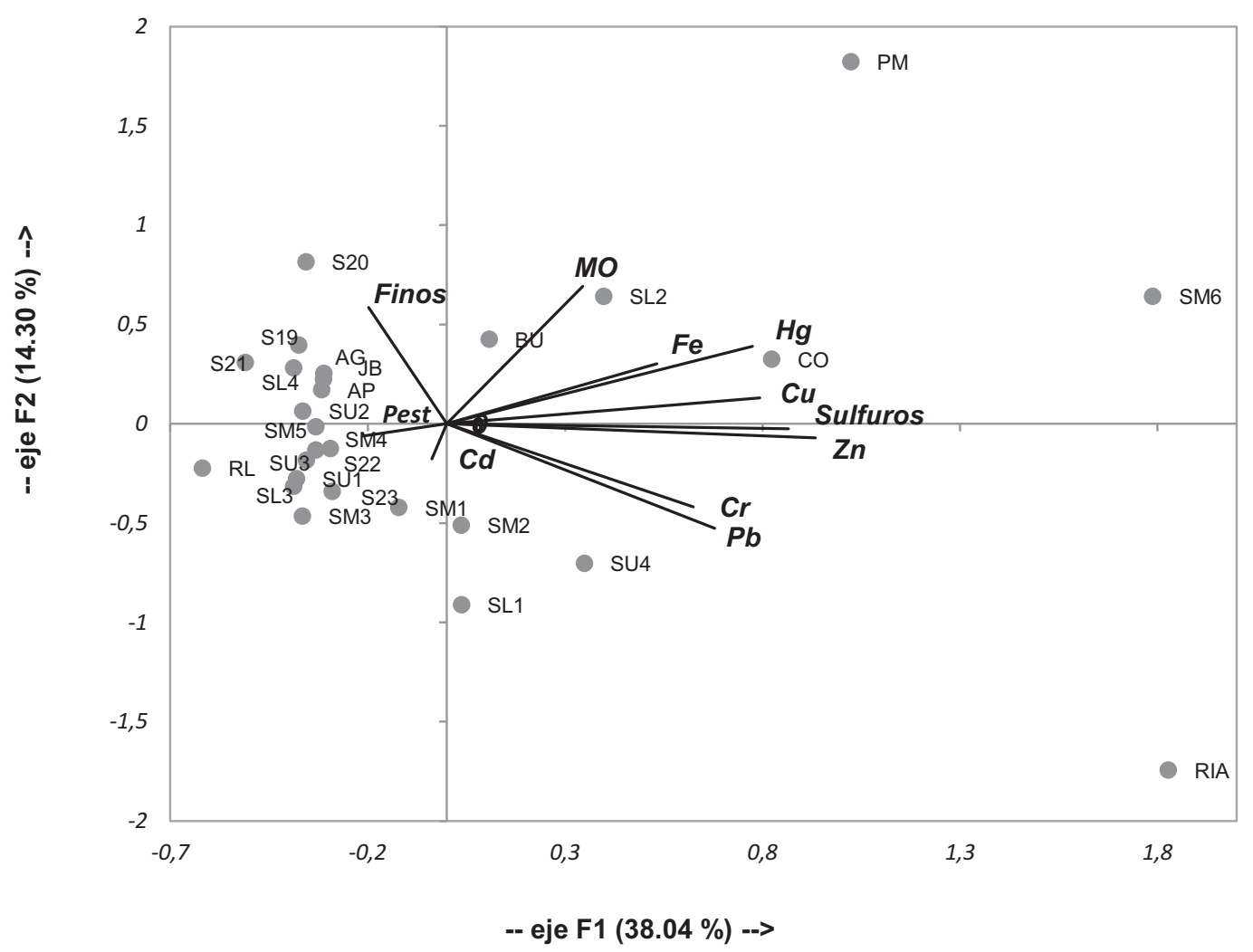

Figura 2. Diagrama del Análisis de Componentes Principales de los dos primeros ejes que explican el $61.4 \%$ de la varianza total correspondiente a los sitios de muestreo. Principal component analysis for the first two components explaining $61.4 \%$ of the total variance of the studied sites. 
y $\mathrm{CO}$ y en menor medida en dos muestras sobre el Río Luján (SU-4 y SL-2); también fue positivo pero con un valor muy bajo, en el sitio correspondiente a la costa uruguaya (BU). El F2 presentó valores de coordenadas positivos principalmente en la muestra PM, BU, SL-2, SM-6 y CO. De esta forma también se puede visualizar la relación entre las variables químicas y las respuestas biológicas observadas en cada muestra. Las muestras que presentaron valores positivos elevados para el F1 y F2 coinciden con las que generaron mayores respuestas tóxicas, sin embargo el único factor que correlaciona negativamente con la supervivencia es el $\mathrm{F} 1$, lo cual indicaría que las variables representadas por el F2 (MO principalmente) no contribuyen significativamente a la toxicidad observada. Las muestras RL, BU y SM6 presentaron valores elevados en el F3, el cual solo estaría representado por la variable plaguicidas totales.

\section{Índice de calidad de sedimentos}

La figura 4 muestra los resultados del SQI calculados para las muestras del Río de la Plata. En la figura se indican las distintas categorías de calidad prefijadas. Considerando los resultados del índice calculado con los valores de ISQG, los sitios RL, PM, CO y RIA se clasifican dentro de la categoría de menor calidad (muy peligroso) de sus sedimentos; mientras que los correspondientes a BU, S19 y S23 se incluyeron en la categoría peligroso. Únicamente los sedimentos provenientes del cauce principal del Río Paraná S22 y S21 se categorizaron como no peligrosos. El resto de las muestras se incluyeron en una categoría intermedia-baja de calidad de sus sedimentos. Cuando se calculó el índice utilizando los valores PEL (los cuales contemplan niveles a los cuales es probable encontrar efectos adversos para la biota acuática), los sitios RIA y CO presentaron

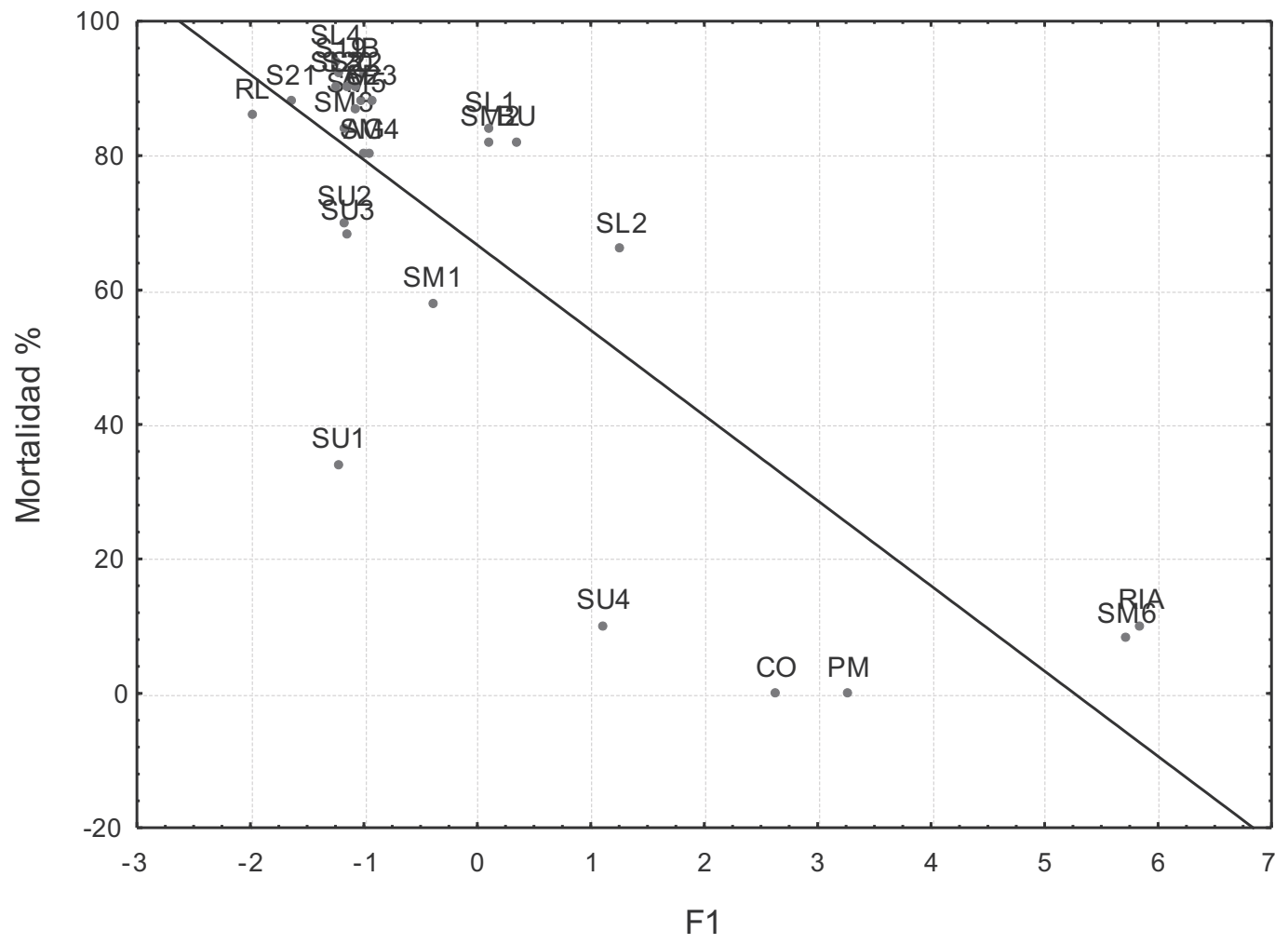

Figura 3. Relación entre el factor 1 del ACP y la supervivencia de H. curvispina en exposiciones de 10 días a los sedimentos estudiados. Relationship between the first axis of the PCA and $\mathrm{H}$. curvispina survival after $10 \mathrm{~d}$ sediment exposure. 


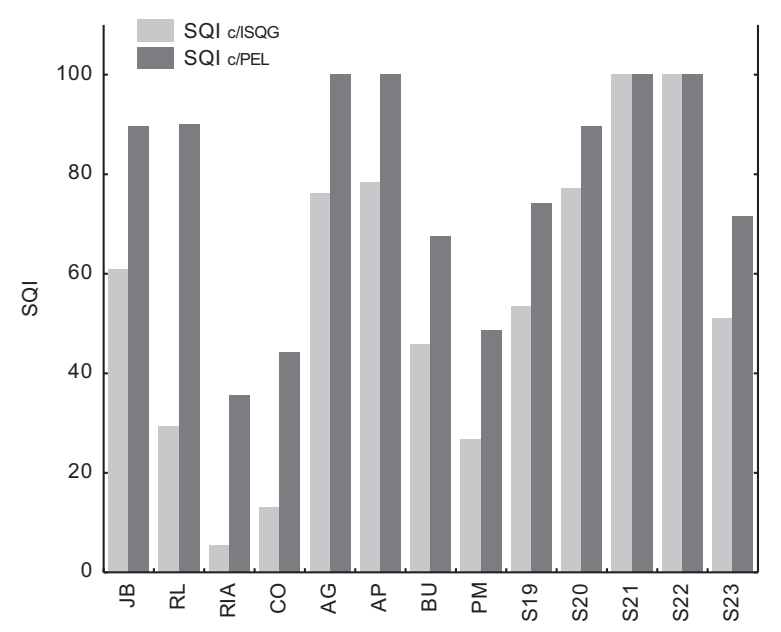

Figura 4. Valores del SQI calculados para los sitios correspondientes a zonas costeras del Río de la Plata. SQI values calculated for the sites located at the Rio de la Plata coast.

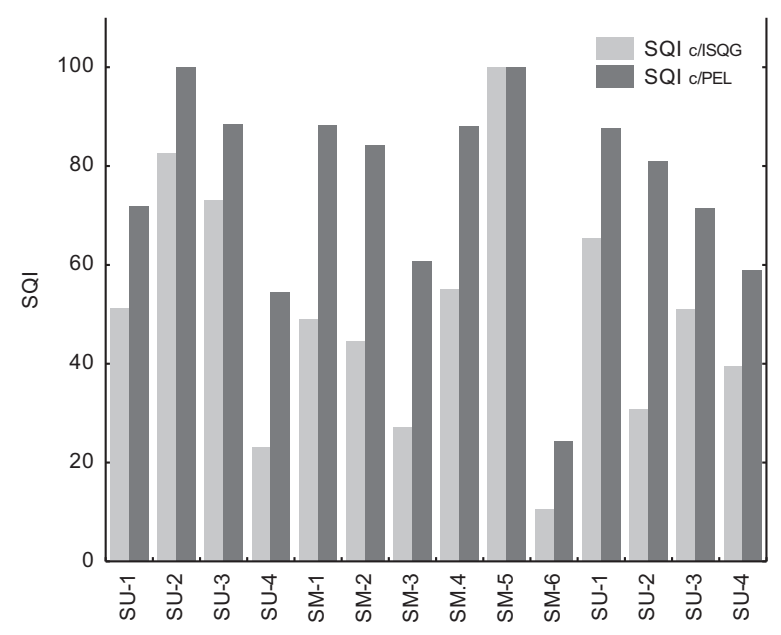

Figura 5. Valores del SQI calculados para los sitios de estudio del Río Luján. SQI values calculated for the studied sites of the Luján River. la menor calidad de materiales, y el PM se incluyó en la categoría peligroso.

En las muestras del Río Luján, los resultados del índice calculado con los valores de ISQG clasifican como muy peligroso la calidad de los sedimentos correspondientes al $43 \%$ de las muestras, mientras que el $30 \%$ se clasificaron como peligrosos (Fig. 5). Por el contrario, cuando se calculó el índice utilizando los valores PEL, una única muestra, SM-6 se mantuvo en la categoría de menor calidad de sus sedimentos, la cual corresponde al sitio aguas abajo del parque industrial mencionado anteriormente.

\section{Índice ecotoxicológico de peligrosidad de sedimentos}

Los resultados de toxicidad obtenidos en los bioensayos con $H$. curvispina se expresaron en un único valor determinado a partir de una clasificación de toxicidad cuya descripción y criterios se detallan en la Tabla 3. En este caso se consideraron más importantes los efectos letales (disminución en la supervivencia) que los subletales (disminución del crecimiento). Esto no significa que los efectos sobre el crecimiento no se consideren como adversos, sino que la reducción en la supervivencia podría resultar en mayores impactos a corto plazo sobre la estabilidad poblacional que la disminución del crecimiento.

En la franja costera del Río de la Plata, a partir de los resultados del IEPS (calculado con ISQG) se incluyen en la categoría de mayor peligrosidad las muestras correspondientes a RL, PM, CO, y RIA; mientras que la muestra S23 se categorizó como peligrosa (Fig. 6). Las S19, S22, AG y BU presentaron una peligrosidad moderada (Fig. 6).

Tabla 3. Criterios adoptados para asignar valores de toxicidad de sedimentos. Adopted reference criteria for assigning sediment toxicity values.

\begin{tabular}{cl}
\hline Valor asignado & Patrones observados en datos de toxicidad \\
\hline 1 & $>50 \%$ de reducción en supervivencia con diferencias significativas con respecto al control \\
6.25 & $>50 \%$ de reducción en punto final subletal con diferencias significativas con respecto al control \\
12.5 & $<20 \%$ de reducción en supervivencia con diferencias significativas con respecto al control \\
25 & $>50 \%$ de reducción en punto final subletal sin diferencias significativas con respecto al control \\
50 & $<20 \%$ de reducción en punto final subletal con diferencias significativas con respecto al control \\
100 & $<20 \%$ de reducción en punto final subletal sin diferencias significativas con respecto al control $<20 \%$ de reducción \\
& en todos los puntos finales. No hay efectos detectables. \\
\hline
\end{tabular}


Los sitios AP, JB, S20 y S21 fueron categorizados como no peligrosos. Los valores del índice calculados con el valor guía correspondiente a los PELs categorizan de la misma forma, como muy peligrosos los sitios PM, CO y RIA.

Para el Río Luján, las muestras de sedimentos de tres sitios fueron categorizadas como mиy peligrosas, SU-1, SU-4 y SM-6; este último corresponde al sitio ubicado aguas abajo de un parque industrial muy importante en la localidad de Pilar (Fig. 7) y presentó un valor muy alto del IEPS en relación a los sitios SU-1 y SU-4. Solo tres de los 14 sitios $(21 \%)$ se incluyeron en la categoría de menor peligrosidad, mientras que el resto (57\%) corresponden a sitios categorizados como peligrosos y probablemente peligrosos.

\section{DISCUSIÓN}

Las metodologías utilizadas (ACP y los dos índices) señalaron como peligrosas a las muestras que presentaron la mayor respuesta tóxica, principalmente se pudieron asociar a la presencia de metales pesados, como de elevados contenidos de sulfuros y materia orgánica presentes en los sedimentos. Considerando que el componente 1 obtenido del ACP, el cual está representado por los metales y sulfuros, correlaciona muy bien (en forma negativa), con la supervivencia de los anfípodos en los bioensayos de toxicidad, esta alternativa es muy útil para clasificar en una primera etapa los sitios estudiados de acuerdo a la peligrosidad de sus sedimentos. En este sentido las muestras correspondientes a RIA, CO, SM6 y PM serían los sitios de mayor peligrosidad en relación a las características químicas y toxicológicas estudiadas. Dado que las respuestas subletales no correlacionaron con las variables analizadas, esta primera clasificación sólo estaría valorando efectos letales agudos, asociados a las muestras más contaminadas.

En la franja costera, el SQI y el IEPS calculados con el ISQG categorizan con la mayor peligrosidad (muy peligroso) los mismos cuatro sitios: CO, RIA, PM y RL, de los cuales los tres primeros coinciden con los sitios de mayor peligrosidad obtenidos con el ACP. La muestra ob-
Tabla 4. Número de sitios que corresponden a cada categoría de peligrosidad con las metodologías propuestas (ACP, SQI, IEPS). Number of sites within each hazard rank with the proposed methodologies (PCA, SQI, IEPS)

\begin{tabular}{lcccccc}
\hline & \multicolumn{5}{c}{ Metodología } \\
Categorías & ACP & SQI & \multicolumn{2}{c}{ IEPS } \\
\hline & & ISQG & PEL & ISQG & PEL Sed Ref \\
\hline Excelente & 10 & 6 & 17 & 7 & 13 & 1 \\
Problemente Peligroso & 7 & 4 & 3 & 9 & 6 & 12 \\
Peligroso & 4 & 7 & 4 & 4 & 3 & 5 \\
Muy Peligroso & 6 & 10 & 3 & 7 & 5 & 8 \\
\hline
\end{tabular}

tenida de la desembocadura del Río Luján (RL), que presenta contenidos detectables de plaguicidas generó respuestas subletales en los anfípodos (Peluso et al., 2013a), mientras que los sedimentos provenientes de la desembocadura del Riachuelo, el puerto de Montevideo y el canal Oeste generaron respuestas letales elevadas (100\% mortalidad) (Peluso et al., 2011) y contenidos de metales que superan en un orden de magnitud los ISQGs para dichos compuestos (Ronco $e t$ al., 2008). A su vez el sitio S23, correspondiente al Río Luján antes de su desembocadura, se categorizó como peligroso solo con el IEPS, dicha muestra también generó respuestas subletales en los bioensayos (Peluso et al., 2013b). Esto indicaría que el IEPS es adecuado para la categorización de peligrosidad de sedimentos que generen tanto respuestas letales como subletales en los anfípodos.

En el caso de la cuenca del Río Luján, hay una mayor diferencia en los resultados de la categorización de calidad entre los dos índices calculados con los niveles ISQGs, debido a que el SQI incluye en la categoría de mayor peligrosidad tres sitios más que el IEPS. Además, el sitio SM2, categorizado como peligroso por el SQI, no presentaría riesgo cuando se calcula el IEPS, esto se debería a la presencia de concentraciones de $\mathrm{Cr}$ total por encima de los niveles guía que presenta dicha muestra, aunque sin evidenciar efectos detectables sobre los organismos. En este sentido, Rifkin et al. (2004), encuentran que la presencia de cromo en mayores concentraciones a los niveles guía en sedimentos no indujeron efectos sobre la supervivencia de los anfípodos expuestos, des- 
tacando la importancia de la valencia en la que se encuentra el metal además de la concentración total presente. Berry et al. (2004) no encontraron buena correlación entre la concentración de cromo total en presencia de sulfuros por encima de $3.4 \mathrm{~g} / \mathrm{Kg}$ y la toxicidad en anfípodos, sin embargo estas concentraciones de sulfuros indican un sedimento de un ambiente anóxico, por lo cual el cromo se encontraría en su estado de valencia $\mathrm{Cr}$ (III) de menor toxicidad.

Cuando se analiza la relación entre los dos índices utilizados y la toxicidad obtenida en los bioensayos, se obtiene una relación positiva significativa $\left(r^{2}=0.8422 ; r=0.9177 / p=0.0000\right.$; $y=-1.1098+0.4948 * x$ ) para el IEPS (Fig. 8), mientras que para el SQI se obtiene un coeficiente de correlación muy bajo $\left(r^{2}=0.3092\right.$; $r=-0.5560 / p=0.0026 ; y=93.6759-5.4097$ $* x)$. Los resultados del IEPS mostraron que el $100 \%$ de las muestras categorizadas como peligrosas presentaron respuestas tóxicas (tanto letales como subletales), mientras que solo un $17 \%$ se categorizaron como no peligrosas y evidenciaron algún grado de toxicidad. Esta situación podría asociarse a falsos negativos, atribuible a factores que generen toxicidad en los anfípodos y la presencia de contaminantes que no fueron evaluados en los estudios. Por otra parte, según el SQI de la totalidad de los sitios categorizados como no peligrosos, el $35 \%$ mostraron respuestas tóxicas para los anfípodos expuestos, mientras que los sitios categorizados como peligrosos el $38 \%$ no fueron tóxicos. Esto último podría deberse a que dicho índice no contempla respuestas biológicas, por lo tanto no tiene en cuenta la biodisponibilidad de los contaminantes en los sedimentos analizados. A partir de los niveles guía de calidad de sedimentos es difícil inferir las relaciones causa-efecto entre los contaminantes y las respuestas tóxicas observadas (Batley et al., 2002; Burton, 2002), por lo cual es aconsejable la utilización de diferentes LEDs. En este sentido, la evaluación de cada LDE se realiza a partir de diferentes análisis o ensayos.

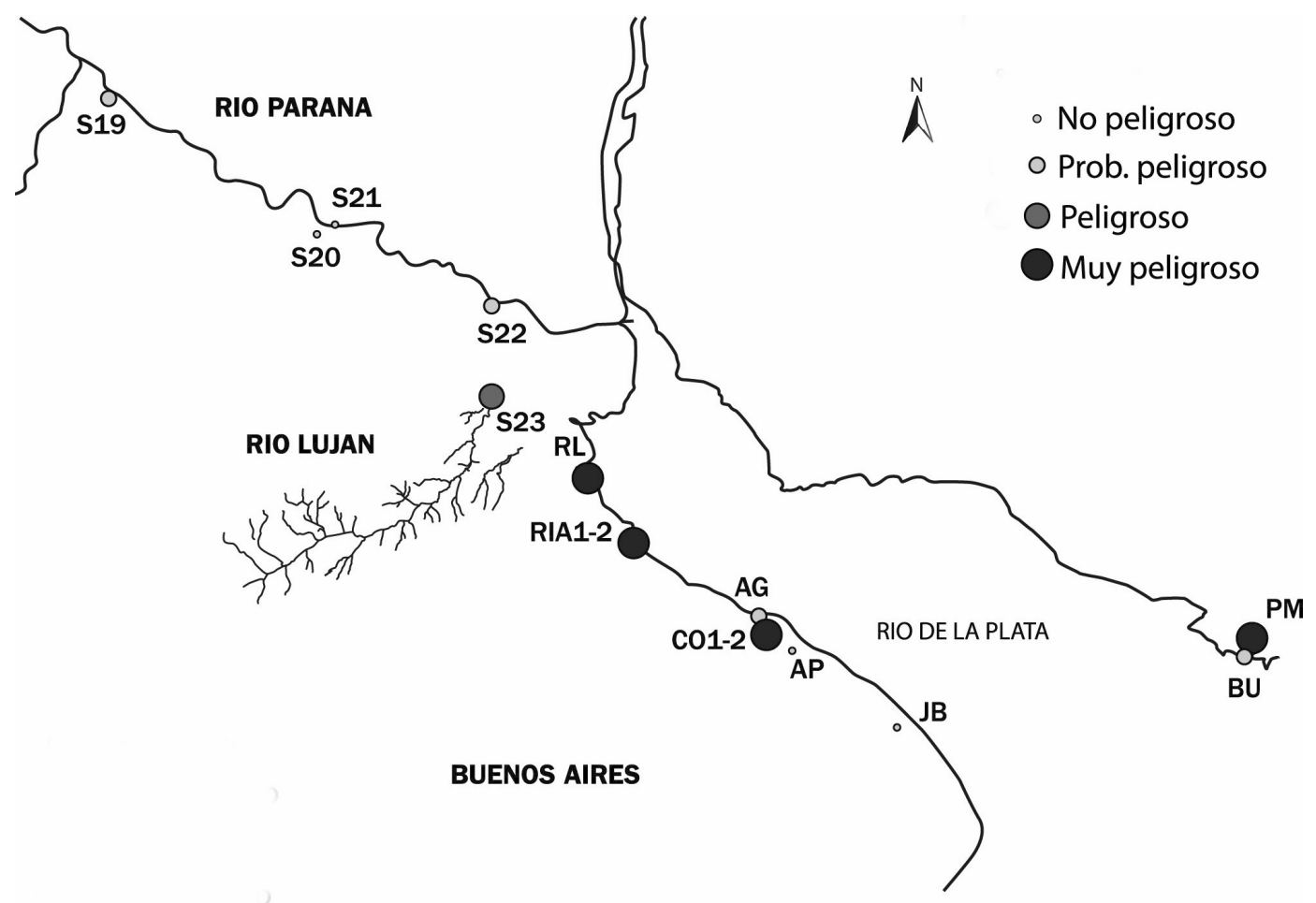

Figura 6. Categorización de peligrosidad de los sedimentos correspondientes a los sitios de la costa del Río de la Plata a partir del cálculo del IEPS. IEPS hazard categorization of sediment samples from the Río de la Plata coast. 


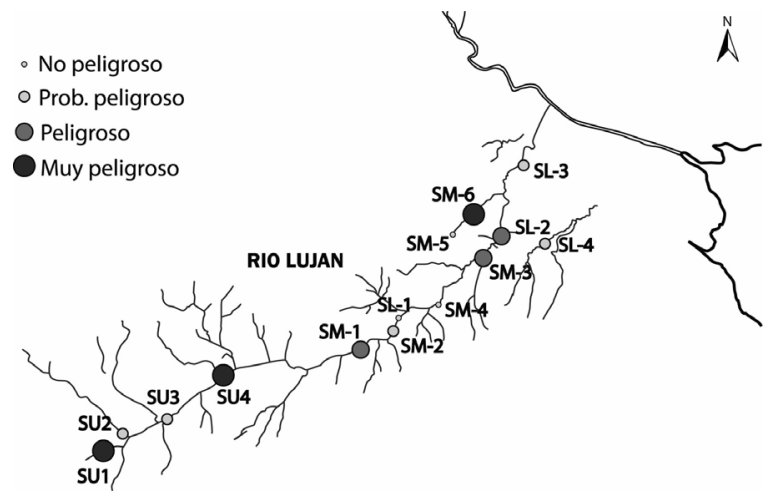

Figura 7. Categorización de peligrosidad de los sedimentos correspondientes al Río Luján a partir del cálculo del IEPS. IEPS hazard categorization of sediment samples from the Rio Luján.

Los resultados provenientes de los diferentes análisis se deben integrar para obtener un único resultado representativo para cada LDE, como por ejemplo índices con datos químicos (Grapentine et al., 2002), sistemas de categorización para resultados toxicológicos (Chapman et al., 2002) o índices bióticos (McPherson et al., 2008; Peluso et al., 2013b).

En la tabla 4 se muestran el número de sitios en cada categoría (peligrosidad de sedimentos) comparando las metodologías utilizadas en este trabajo. Los índices calculados a partir de los ISQGs permiten categorizar de forma más conservativa que utilizando los valores PEL, ya que estos últimos son valores de efecto probable sobre la biota. Comparando los índices SQI e IEPS (utilizando ISQGs), el primero categoriza mayor cantidad de sitios como muy peligrosos, sin embargo cuando se utilizan PELs la relación entre los dos índices se invierte y el IEPS categoriza mayor número de muestras como peligrosas y muy peligrosas. Es decir, utilizando datos toxicológicos en el cálculo del índice IEPS, la diferencia entre el número de sitios calculados con los dos niveles de referencia antes mencionados, es menor que utilizando el SQI (no incluye información toxicológica). La cantidad de sitios en las diferentes categorías con el IEPS (ISQGs) son similares utilizando ACP e IEPS calculado con los valores de la muestra del sitio control.

El esquema incluido en la figura 9 resume una propuesta de etapas a seguir en el diagnóstico de

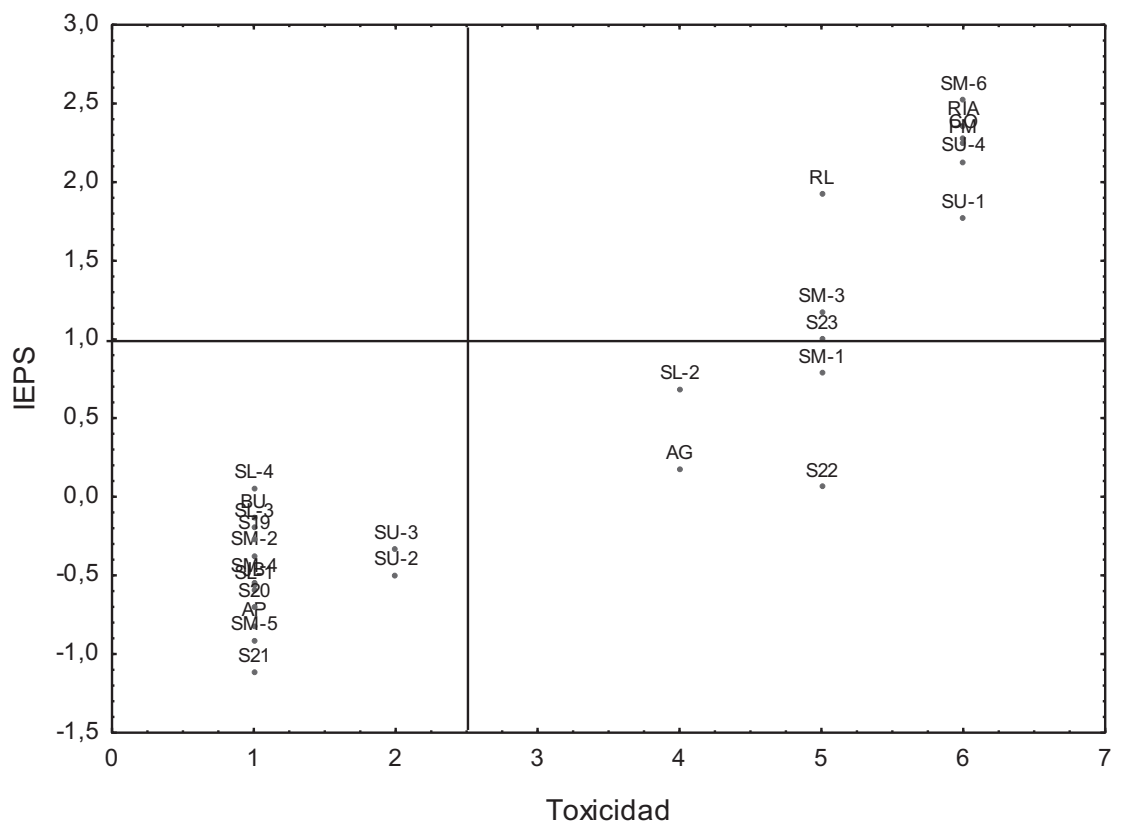

Figura 8. Relación entre valores de toxicidad y el índice IEPS. Relationship between toxicity and IEPS values. 


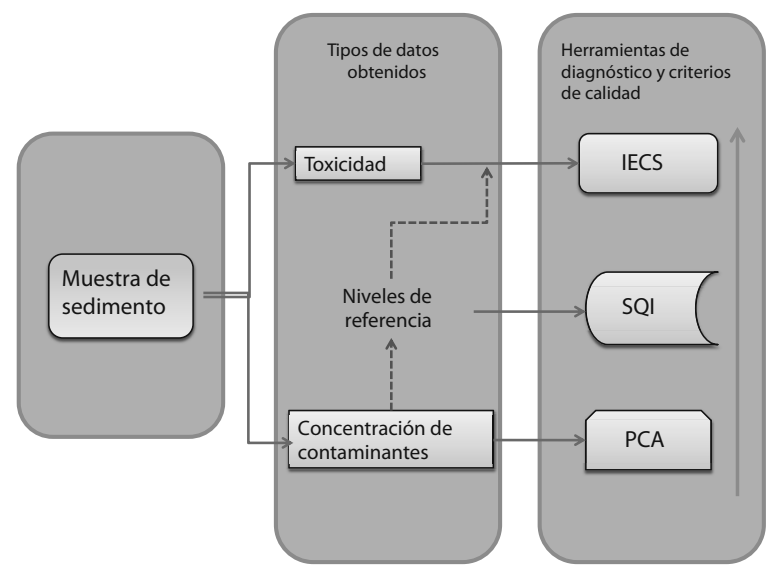

Figura 9. Esquema de aplicación de herramientas para el diagnóstico de calidad de sedimentos. Follow up procedure for the application of the proposed methodologies.

calidad de sedimentos en función de la estrategia a adoptar y tipo de datos (concentraciones, toxicidad, niveles de referencia). Se podría partir de una estrategia que utilice únicamente concentraciones de compuestos químicos sobre las muestras de sedimentos, proponiéndose en este caso un ACP. Complementariamente, si se cuenta con información de niveles de referencia, la obtención de un índice como el SQI es de utilidad en la clasificación de los sitios estudiados. Además de la caracterización físico-química de las muestras, se recomienda una evaluación toxicocológica de los sedimentos, lo cual permite, a partir de ambos tipo de datos, construir un índice IEPS que incluye mayores líneas de evidencia en la toma de decisiones en planes de gestión del recurso. Por otra parte, a partir del ACP y las respuestas biológicas se recomienda estudiar la relación entre los factores principales que caracterizan los sedimentos y las respuestas observadas.

De acuerdo al análisis realizado a partir de las metodologías de diagnóstico evaluadas, se recomienda la utilización de índices que contemplen la caracterización química de la matriz y los efectos biológicos sobre la biota expuesta como el IEPS, además de considerarse niveles de referencia propios de la región de estudio, que muestren escenarios realistas de interacción tóxico-matriz, que permita tener en cuenta la biodisponibilidad de los materiales estudiados (Chapman, 2007). En relación a los efectos sobre la biota, cuan- do fuera necesario contar con valoraciones más complejas se podrían incorporar líneas de evidencia basadas en bioensayos a largo plazo para evaluar efectos crónicos, además de baterías de bioensayos con mayor número de especies que representen diferentes niveles tróficos y/o rutas de exposición (Blaise \& Férard, 2005).

\section{AGRADECIMIENTOS}

El presente estudio se realizó con financiamiento del proyecto PIP-0410 del Consejo Nacional de Investigaciones Científicas y Técnicas -CONICET-. Los autores agradecen los valiosos comentarios y sugerencias de los revisores del manuscrito.

\section{BIBLIOGRAFÍA}

ABESSA, D. M. S., R. S. CARR, E. C. P. M. SOUZA, B. R. RACHID, L. P. ZARONI, Y. A. PINTO, M. R. GASPARRO \& P. M. MACIEL. 2008. Integrative ecotoxicological assessment of a complex tropical estuarine system. Marine Pollution: New Research. Nova Science Publishers, Inc, New York, USA.

ALEXANDER, M. 2000. Critical review aging, bioavailability, and overestimation of risk from environmental pollutants. Environmental Science and Technology, 34: 4259-4265.

ANDRADE, M. I. 1986. Factores de deterioro ambiental en la cuenca del Río Luján. Buenos Aires: Contribución del Instituto de Geografía, Facultad de Filosofía y Letras (UBA).

ANGUIANO, O. L., A. FERRARI, J. SOLEÑO, M.C. MARTÍNEZ, A. VENTURINO, A. M. PECHEN DE D'ANGELO \& C. M. MONTAGNA. 2008. Enhanced esterase activity and resistance to azinphosmethyl in target and nontarget organisms. Environmental Toxicology and Chemistry, 27: 21172123.

BATLEY, G. E., G. A. BURTON, P. M CHAPMAN \& V. E. FORBES. 2002. Uncertainties in sediment quality Weight-of- Evidence (WOE) assessments. Human and Ecological Risk Assessment, 8:15171547.

BERRY, W. J., W. S. BOOTHMAN, J. R. SERBST \& P. A. EDWARDS. 2004. Predicting the toxicity of 
chromium in sediments. Environmental Toxicology and Chemistry, 23: 2981-2992.

BLAISE, C. \& J. F. FÉRARD. 2005. Overview of contemporary toxicity testing. In: Small-scale Freshwater Toxicity Investigations. (Volume 2, Hazard Assessment Schemes). C. Blaise \& J. F. Férard (eds.): 1-68., Springer Publishers, Dordrecht, The Netherlands.

BOMBARDIER, M. 2005. The SED-TOX index for toxicity assessment of contaminated solid matrices. In: Small-scale Freshwater Toxicity Investigations. (Volume 2, Hazard Assessment Schemes). C. Blaise \& J. F. Férard (eds.): 1-68. Springer Publishers, Dordrecht, The Netherlands.

BRIANO, L., F. FRITZCHE \& M. VIO. 2003. El lugar de la industria. Parques industriales de la Región Metropolitana. Revista Eure, 29, 109-135.

BURTON, G. A. 2002. Sediment quality criteria in use around the world. Limnology, 3: 65-75.

CAMILIÓN, M. C., M. J. MANASSERO, M. A. HURTADO \& A. E. RONCO. 2003. Copper, lead and zinc distribution in soils and sediments of the South Western coast of the Río de la Plata Estuary. Journal of Soils and Sediments, 3: 213-220.

CEQG 2002. Summary of Existing Canadian Environmental Quality Guidelines. www.ccme.ca/assets/ pdf/e1_06.pdf

CESAR, A., D. MOLEDO, D. S. ABESSA, C. DIAS, S. PEREIRA, R. SANTOS, N. FERNÁNDEZ, R. B. CHOUERI \& T. A. DELVALLS. 2009. A simple approach to integrate the ecotoxicological and chemical data for the establishment of environmental risk levels. Brazilian Archives of Biology and Technology, 52: 233-240.

CHAPMAN, P. M., B. G. MCDONALD \& G. S. LAWRENCE. 2002. Weight-of-evidence issues and frameworks for sediment quality (and other) assessments. Human Ecological Risk Assessment, 8: 1489-1515.

CHAPMAN, P. M. 2007. Determining when contamination is pollution-Weight of evidence determinations for sediments and effluents. Aquatic Sciences, 33: 492-501.

CORREIA, A. D., G. LIMA, M. H. COSTA, \& D. R. LIVINGSTONE. 2002. Studies on biomarkers of copper exposure and toxicity in Gammarus locusta: Induction of metallothionein and lipid peroxidation. Biomarkers, 7: 101-127.

COSTA, F. O., A. D. CORREIA \& M. H. COSTA. 1998. Acute marine sediment toxicity: A potential new test with the amphipod Gammarus locusta.
Ecotoxicology and Environmental Safety, 40: 8187.

GARCÍA, M. E., A. RODRIGUES-CAPÍTULO. \& L. FERRARI. 2010. Age-related differential sensitivity to cadmium in Hyalella curvispina (Amphipoda) and implications in ecotoxicity studies. Ecotoxicology and Environmental Safety, 73: 771-778.

GIUSTO, A. \& L. FERRARI. 2008. Copper toxicity on juveniles of Hyalella pseudoazteca Gonzalez and Watling, 2003. Bulletin of Environmental Contamination and Toxicology, 81: 169-173.

GIUSTO, A., L. A. SOMMA \& L. FERRARI. 2012. Cadmium toxicity assessment in juveniles of the Austral South America amphipod Hyalella curvispina. Ecotoxicology and Environmental Safety, 79: $163-169$.

GIUSTO, A., A. SALIBIÁN \& L. FERRARI. 2014. Biomonitoring toxicity of natural sediments using juvenile Hyalella curvispina (Amphipoda) as test species: evaluation of early effect endpoints. Ecotoxicology, 23: 293-303.

GRAPENTINE, L., C. MARVIN \& S. PAINTER. 2002. Initial development and evaluation of a Sediment quality Index for the Great Lakes Region. Human and Ecological Risk Assessment, 8: 1549-1567.

GUICHÓN, M. L., M. ANGELINI, A. BENITEZ, C. SERAFINI \& M. H. CASSINI. 1999. Caracterización ambiental de la cuenca del río Luján. Revista de Teledetección (España), 11: 5-12.

HILL, M. K. 2010. Understanding environmental pollution. Cambridge University Press.

HYLAND, J. L., W. L. BALTHIS, V. D. ENGLE, E. R. LONG, J. F. PAUL, J. K. SUMMERS \& R. F. VAN DOLAH. 2003. Incidence of stress in benthic communities along the U.S. Atlantic and Gulf of Mexico coasts within different ranges of sediment contamination from chemical mixtures. Environmental Monitoring and Assessment, 81: 149_ 161.

IADC/CEDA 1997. Environmental aspects of dredging-conventions, codes and conditions: marine disposal. International Association of Dredging Companies (IADC), \& Central Dredging Association (CEDA), Netherlands, 1-71.

INGERSOLL, C. G. 1995. Sediment Test. In: Fundamentals of aquatic toxicology. G. M. Rand (ed.) Taylor \& Francis, Washington, DC.

KREBS, F. 2005. The pt-method as a hazard assessment scheme for sediments and dredged material. In: Small-scale Freshwater Toxicity Investigations. 
(Volume 2, Hazard Assessment Schemes). C. Blaise \& J. F. Férard (eds.): 1-68. Springer Publishers, Dordrecht, The Netherlands.

KREIMER, E. D., D. E. PALACIOS \& A. E. RONCO. 1996. A proposal for dredging contaminated sediments at the Dock Sud Port, Argentina. Proceedings of the International Symposium on Coastal Ocean Space Utilization, COSU'96, 435-445, Buenos Aires.

LANDRUM, P. F. \& J. A. ROBBINS. 1990. Bioavailability of sediment-associated contaminants to benthic invertebrates. In Sediments: Chemistry and Toxicity of In-Place Pollutants. R. Baudo, J. P. Giesy \& H. Muntau (eds.): 237-263. Lewis, Ann Arbor, MI, USA.

LOSSO, C., A. ARIZZI NOVELLI, M. PICONE, D. MARCHETTO, G. PESSA, E. MOLINAROLI, P. F. GHETTI \& A VOLPI GHIRARDINI. 2004. Evaluation of surficial sediment toxicity and sediment physicochemical characteristics of representative sites in the Lagoon of Venice (Italy). Journal of Marine Systems, 51: 281-292.

MCPHERSON, C., P. M. CHAPMAN, A. M. H. DEBRUYN \& L. COOPER. 2008. The importance of benthos in weight of evidence sediment assessments -a case study. Science of the total environment, 394: 252-64.

MUGNI, H., A. RONCO \& C. BONETTO. 2011. Insecticide toxicity to Hyalella curvispina in runoff and stream water within a soybean farm (Buenos Aires, Argentina). Ecotoxicology and Environmental Safety, 74: 350-354.

NEUPARTH, T., A. D. CORREIA, F. O. COSTA \& M. H. COSTA. 2005. Multi-level assessment of chronic toxicity of estuarine sediments with the amphipod Gammarus locusta: I. Biochemical endpoints. Marine Environmental Research, 60: 6991.

PELUSO, L., A. GIUSTO, G. D. BULUS ROSSINI, L. FERRARI, A. SALIBIÁN \& A.RONCO. 2011. Hyalella curvispina (Amphipoda) as a test organism in laboratory toxicity testing of environmental samples. Fresenius Environmental Bulletin, 20: 372-376.

PELUSO, M. L., G. BULUS ROSSINI, A. SALIBIÁN \& A. RONCO. 2013a. Physicochemical and ecotoxicological based assessment of bottom sediments from the Luján River basin, Buenos Aires Argentina. Environmental Monitoring and Assessment, 185: 5993-6002.
PELUSO, L., M. ABELANDO, C. A. APARTíN, P. ALMADA \& A. RONCO. 2013b. Integrated ecotoxicological assessment of bottom sediments from the Paraná basin, Argentina. Ecotoxicology and Environmental Safety, 98: 179-186.

QUINN, G. P. \& M. J. KEOUGH. 2002 Experimental Design and Data for Biologists. Cambridge University Press. Cambridge.

RAND, G. M., P.G. WELLS \& L. MCCARTY. 1995 Introduction to aquatic toxicology. In: Fundamentas of Aquatic Toxicology. G. M. Rand (ed.): 3-66. Taylor \& Francis, Washington, DC.

RIBA, I., M. FORJA, A. GOMEZ-PARRA \& T. DELVALLS. 2004. Sediment quality in littoral regions of the Gulf of Cadiz: a triad approach to address the influence of mining activities. Environmental Pollution, 132: 341-353.

RIFKIN, E., P. GWINN \& E. BOUWER. 2004. Chromium speciation indicates toxicity of benthic organisms better than total chromium does. Environmental Science and Technology, 15: 267-271.

RONCO, A. E., M. C. SOBRERO, P. R ALZUET, G. BULUS ROSSINI \& B. DUTKA. 1995. Screening for sediment toxicity in the Río Santiago basin: A base line study. Environmental Toxicology and Water Quality, 10: 35-39.

RONCO,A. E., M. C. CAMILIÓN \& M. J. MANASSERO. 2001. Geochemistry of heavy metals in bottom sediments from streams of the western coast of the Río de la Plata estuary, Argentina. Environmental Geochemistry and Health, 23: 89103.

RONCO, A. E., M. L. PELUSO, M. JURADO, G. BULUS ROSSINI \& A. SALIBIÁN. 2008. Screening of sediment pollution in tributaries from the southwestern coast of the Río de la Plata estuary. Latin American Journal of Sediment Basin Analysis, 15: 129-142.

SPROVIERI, M., M. L FEO, L. PREVEDELLO, D. S MANTA, S. SAMMARTINO, S. TAMBURRINO \& E. MARSELLA. 2007. Heavy metals, polycyclic aromatic hydrocarbons and polychlorinated biphenyls in surface sediments of the Naples harbour (Southern Italy). Chemosphere, 67: 998-1009.

USEPA. 2000. Methods for measuring the toxicity and bioaccumulation of sediment-associated contaminants with freshwater invertebrates. Second Edition. U.S. Environmental Protection Agency. Office of Science \& Technology Office of Water. Report: EPA 600/R-99/064. 
WALKER, C. H., S. P. HOPKIN, R. M. SIDLY \& D. B. PEAKALL. 2006. Principles of ecotoxicology. CRC Press,Taylor \& Francis Group. Boca Raton, Florida.

WENNING, R. J., C. G. INGERSOLL, G. BATLEY \& D. W. MOORE. 2004. Use of Sediment Quality
Guidelines (SQGS) and Related Tools for the Assessment of Contaminated Sediments. SETAC Press (Society of Environmental Toxicology \& Chemistry): Pensacola, FL.

ZAR, J. H. 2010. Biostatistical Analysis. PrenticeHall, New Jersey. 